\title{
Magnetoresistance of single-domain ferromagnetic particles
}

\author{
J. Aumentado and V. Chandrasekhar \\ Department of Physics and Astronomy, Northwestern University, Evanston, IL 60208
}

\begin{abstract}
We have performed magnetoresistance measurements on single-domain, submicron elliptical $\mathrm{Ni}$ particles using nonmagnetic probes in a four probe geometry at liquid helium temperatures. In the smallest particles, the magnetoresistance shows sharp jumps which are associated with the switching of individual domains. Using an anisotropic magnetoresistance model, we can reconstruct hysteresis loops of the normalized magnetization. The remanent magnetization in zero applied magnetic field is typically 15 percent less than the saturation magnetization. This relaxation of the magnetization may be due to surface effects or crystal grain structure in the particles.
\end{abstract}

The study of micromagnetics is currently experiencing a revival as experimental techniques improve to the point where it is possible to observe the magnetization dynamics of single submicron-sized particles. While the methods for studying single particle micromagnets have ${ }^{\mathrm{So}}$ far been limited to magnetoforce microscopy (MFM) 14 Loreptr microscopy 2 and microbridge SQUID magnetometry, 3 transport-based micromagnetic measurements may provide a robust alternative which is viable over a large temperature scale. Until recently, experimental efforts at studying electrical transport in mesoscale magnets have mostly concentratecon arrays of thin wires, 6 arrays of chains of particles, 0 and very long, thin single wires.l However, future mesoscopic spin-transport devices are likely to incorporate singledomain ferromagnetic particles in combination with nonmagnetic elements. Consequently, it is important that the transport characteristics of single-domain particles be well understood.

In this Letter, we report on our measurements of the magnetoresistance of patterned submicron single-domain elliptical $\mathrm{Ni}$ thin films in magnetic fields in the plane of the films. At low fields, a hysteretic magnetoresistance is observed, similar to that seen by Hong and Giordano in long $\mathrm{Ni}$ wires. $\mathrm{g}$ In addition, a single discrete jump or a series of discrete jumps can also be observed which are associated with magnetization switching in the domains of the particle. Using an anisotropic magnetoresistance (AMR) model, 10 we can deduce the normalized magnetization of the particles as a function of magnetic field. The resulting hysteresis loops show that the remanent magnetization is smaller than the saturation magnetization, which may be due to surface effects or misalignment of the direction of magnetization in the crystal grains of the particle.

Figure 1 shows scanning electron micrographs of one of our typical samples, along with a schematic illustrating the device structure. The samples were fabricated onto an oxidized Si substrate using conventional bilayer e-beam lithography and liftoff techniques in two steps. In the first step, the elliptical particles were patterned and deposited so that they lay flat on the substrate. Ni (99.995\% pure, $30 \mathrm{~nm}$ thick) was deposited in an e-gun

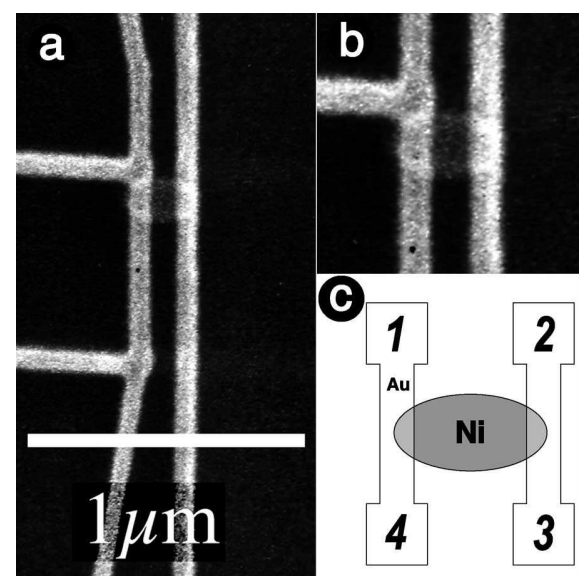

FIG. 1. SEM and schematic representations of devices: (a) typical probe and magnet configuration, (b) enlargement of Ni ellipse, (c) schematic of four probe measurement.

evaporator at a base pressure of $5 \times 10^{-8}$ Torr at a rate of $0.01 \mathrm{~nm} / \mathrm{s}$, resulting in films with a resistivity of approximately $8.3 \mu \Omega-\mathrm{cm}$ at $4.2 \mathrm{~K}$, as measured on a coevaporated $1 \mu \mathrm{m}$ wide $\mathrm{Ni}$ thin film. The elliptical geometry constrained the easy axis in-plane along the major axis of the ellipse. The series of particles discussed here had lateral dimensions of either $120 \mathrm{~nm} \times 240 \mathrm{~nm}$ or $220 \mathrm{~nm} \times 640 \mathrm{~nm}$. This size was chosen because particles of similar dimensions measured by microbridge SQUID magnetometry have demonstrated sharp, single magnetization switches and nominally square hysteresis loops in fields applied along the easy axis. 3 In the second lithography step, Au probes were patterned to make electrical contact to the particle. Prior to the $\mathrm{Au}$ deposition, the exposed $\mathrm{Ni}$ contact areas were cleaned using an ac $\mathrm{Ar}^{+}$etch. Au (99.999\% pure, $60 \mathrm{~nm}$ thick) was then deposited by e-gun evaporation, creating two narrow (70 nm width) nonmagnetic probe wires extending across the ellipse along the minor axis direction. Four terminal measurements using these probes allowed us to measure the resistance of the ferromagnetic particle, along with a small contribution from the magnetic/nonmagnetic interface. 
The use of nonmagnetic probes is a unique and essential component of our sample design. The only other transport experiments in particles of this size range of which we are aware usedprobes fabricated from the same material as the sample.11 This configuration is problematic since the magnetic probes will modify the magnetization of the sample. As the probe size becomes comparable to the sample size, this effect will become more pronounced. In addition, the injection of spin-polarized carriers from the magnetic probes into the magnetic particle might also be expected to affect the magnetoresistance.12 We avoid these difficulties by eliminating the ferromagnetic probes and using nonmagnetic $\mathrm{Au}$ probes to make contact to our devices.

The samples were measured in a pumped ${ }^{4} \mathrm{He}$ cryostat with the major axis of the ellipses aligned parallel or perpendicular (to within $5^{\circ}$ ) to the applied magnetic field direction. The resistance was measured in a fourprobe configuration using standard ac lock-in/bridge techniques with excitation currents between 0.1 and 5.0 $\mu \mathrm{A}$ at $11.7 \mathrm{~Hz}$ at temperatures between 1.2 and $10 \mathrm{~K}$. The traces were identical at all excitation currents in this range, with the exception of an improved signal-to-noise ratio at higher currents. In this probe configuration the direction of the current is primarily along the major axis of the ellipse.
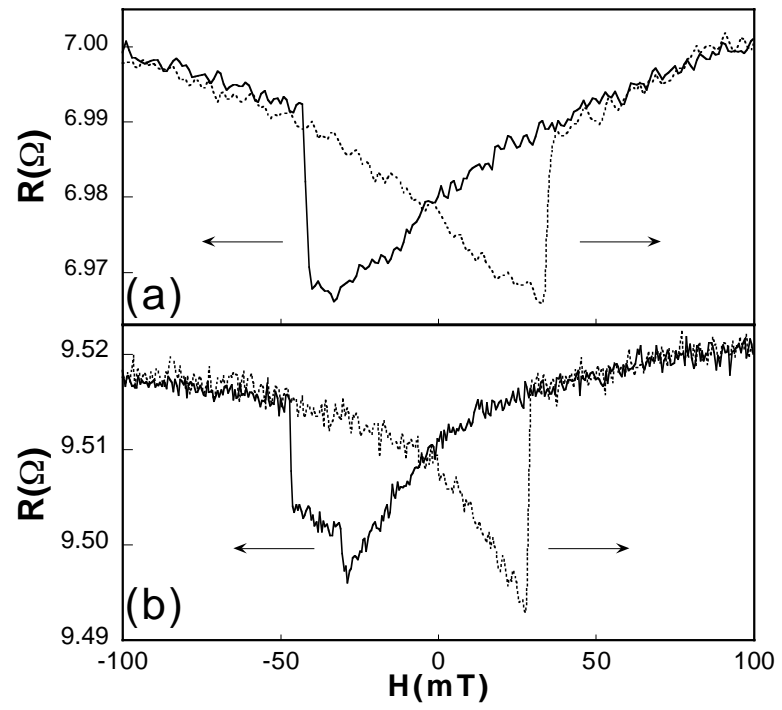

FIG. 2. Parallel field magnetoresistance traces of Ni ellipses at $\mathrm{T}=1.5 \mathrm{~K}$. (a) $120 \mathrm{~nm} \times 240 \mathrm{~nm} \mathrm{Ni}$ ellipse. $220 \mathrm{~nm} \times 640 \mathrm{~nm}$ ellipse.

Figure 2(a) shows a typical low-field parallel magnetoresistance trace taken at $T=1.5 \mathrm{~K}$ in a $120 \mathrm{~nm} \times 240 \mathrm{~nm}$ elliptical particle. The magnetic field is swept between $\pm 2000 \mathrm{G}$ at a constant rate of less than $2 \mathrm{G} / \mathrm{s}$. Following the sweep from positive-to-negative magnetic field (solid trace), one can see a single discrete jump in the magnetoresistance, while the negative-to-positive sweep (dotted trace) shows similar behavior in the opposite direction. Before the jumps, the magnetoresistance is completely reversible. After the jumps, the traces then become reversible on a second curve which is nearly mirror symmetric with the original curve. In other samples, the magnetization reversal may occur in multiple steps. Figure 2(b) shows similar traces for a $220 \mathrm{~nm} \times 640 \mathrm{~nm}$ elliptical particle. The negative-to-positive field sweep (dotted line) shows a single jump as before, but two distinct jumps are observed in the opposite sweep direction (solid line). The single large jump in the negative-to-positive sweep indicates a complete switch in the magnetization direction occuring in one step, while the two distinct steps in the reverse direction may be due to an intermediate magnetization state that would be found in the presence of domain structure or magnetization pinning. We note that small positive jumps in the magnetoresistance have also been seen in long wires by Hong and Giordano in studies of domain wall scattering 9

These results demonstrate that one might infer the magnetic state of a single-domain particle by measuring its magnetoresistance. In order to do this, one needs to understand the mechanism which gives rise to the magnetoresistance. Hong and Giordanol attributed the magnetoresistance in their $\mathrm{Ni}$ wires to scattering of electrons by magnons. However, in our samples, the temperature independence of the magnetoresistance is inconsistent with this explanation. Shearwood et al.5 proposed instead that the similar magnetoresistance behavior they observed in their arrays of permalloy wires arose from the well-known anisotropic magnetoresistance (AMR) of ferromagnets.10 The AMR of ferromagnets is due to an anisotropic spin-orbit interaction which leads to a resistance sensitive to the angle between the current density and the magnetization. The resistivity due to AMR can be expressed as 10

$$
\rho=\rho_{\perp}+\Delta \rho_{A M R} \cos ^{2} \xi
$$

where $\Delta \rho_{A M R} \equiv \rho_{\|}-\rho_{\perp}$ and $\xi$ is the angle between the current and magnetization. $\rho_{\|}\left(\rho_{\perp}\right)$ is the extrapolation to zero magnetic induction $(B=0)$ of the magnetoresistance with the magnetic field parallel (perpendicular) to the direction of the current. Experimentally, $\Delta \rho_{A M R}$ is found to be positive, so that the resistance with the magnetization parallel to the current direction is greater than the resistance with the magnetization perpendicular to the current direction. We believe that the magnetoresistance of our particles arises from this effect.

Consider again the magnetoresistance of the single small ferromagnetic particle shown in Fig. 2 in terms of the AMR model. At large negative magnetic fields, the magnetization is saturated in the direction of the field, and the resistance has its highest value, consistent with the AMR model. As the field is increased, i.e., the magntitude of the field is decreased, the resistance of the sample decreases. If we make the assumption that the magnetization throughout the ellipse is uniform and rotates coherently relative to the current direction, this decrease in resistance indicates that the direction of the 
magnetization is changing as a function of field, since the direction and magnitude of the current are constant. We can express the projection of the magnetization along the current direction (or major axis of the ellipse) as $M_{x}=M_{0} \cos \xi$ (where $M_{0}$ is the saturation magnetization). From Eq. (1), the normalized magnetization $m_{x}(H)=M_{x} / M_{0}$ can be expressed in terms of the field dependent resistance $\rho(H)$ as

$$
\left|m_{x}(H)\right|=\left(\frac{\rho(H)-\rho_{\perp}}{\Delta \rho_{A M R}}\right)^{\frac{1}{2}} .
$$

Using this equation, hysteresis loops of the normalized magnetization projection may be reconstructed from the resistance, inserting appropriate signs at the switches.

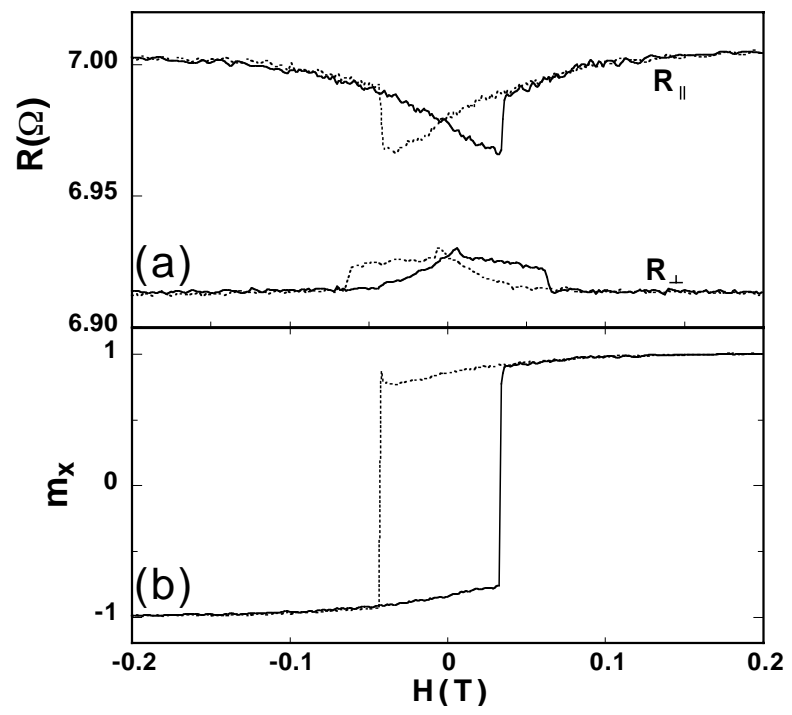

FIG. 3. Magnetization reconstruction from magnetoresistance of $120 \mathrm{~nm} \times 240 \mathrm{~nm}(30 \mathrm{~nm}$ thick $)$ Ni ellipse at $\mathrm{T}=1.5 \mathrm{~K}$. (a) parallel and perpendicular field magnetoresistances (in-plane), (b) reconstructed normalized magnetization, $m_{x}$, from the parallel field magnetoresistance. The solid curve is taken while sweeping the field in the positive direction. The dotted curve is taken in the negative direction.

Figure 3(a) shows the magnetoresistance of the single Ni particle of Fig. 2(a) in magnetic fields both perpendicular and parallel to the major axis of the ellipse, with the current along the major axis in both cases. From these measurements, one can obtain the AMR ratio, $\Delta \rho_{A M R} / \rho_{\text {ave }}\left(\right.$ where $\left.\rho_{\text {ave }} \equiv\left(\rho_{\|}+2 \rho_{\perp}\right) / 3\right)$, which varies between 1.25 and $1.5 \%$ for our amples. This is somewhat lower than results in bulk Ni10 but is consistent with expectations in specimens of reduced dimensionality 13 Figure 3(b) shows the normalized magnetization obtained from the data of Fig. 3(a) as described above. The magnetization shows clean switches at applied magnetic fields of $\sim \pm 350 \mathrm{G}$, indicative of the presence of a single domain whose magnetization switches direction at these fields. Prior to the switch, however, the magnetization decreases to values less than the saturation magnetization at low fields, dropping to approximately 0.85 of the saturation magnetization at zero field. In the spirit of the coherent rotation model, this would correspond to a misalignment of the magnetization from the major axis of the ellipse of $\sim 20-30^{\circ}$ in the samples we have studied. More generally, a remanence ratio of less than unity might indicate the presence of a nonuniform magnetization in the sample in zero applied field.

What are the possible origins of a nonuniform magnetization in our particles? A number of mechanisms can be suggested. First, the particles are elliptical films, and not ellipsoids of revolution which means that the magnetization in the particle is not uniform. In high magnetic fields, all magnetic moments in the particle would be aligned along the major axis of the ellipse, but at low fields, the moments at the surface of the film in particular would tend to be canted due to the nonuniform demagnetizing field. A rough order of magnitude estimate of this misalignment can be obtained by considering the moments in a rim of width of the thickness of the film around the perimeter of the ellipse to be completely perpendicular to the major axis of the ellipse. With the dimensions of the sample of Fig. 3, this gives an estimate of 0.75 for the remanence ratio, in good agreement with the value of 0.85 we observe. Second, a remanence ratio of less than unity may be due to the polycrystalline nature of the $\mathrm{Ni}$ films. If the small $\mathrm{Ni}$ particle consists of a few crystal grains with randomly oriented crystalline axes, the magnetization of each grain in zero applied field may relax along crystal axes due to crystalline anisotropy. This would lead to reduction in the component of the total magnetization parallel to the major axis of the ellipse. Finally, modification of the spin-orbit interaction close to the surface is also expected to lead to the misalignment of moments near the surface.14 At present, we cannot distinguish between these different mechanisms, but we are inclined towards the ngnuniform demagnetizing field as the simplest mechanism 5 which would account for our observations. We should note that remanence ratios approaching unity have been observed in magnetization measurements on similarly sized particles by Wernsdorfer et al 3 using microbridge SQUIDs, but we believe that these results might be affected by flux exclusion due to the Meissner effect in the superconducting microbridge devices. There are no nearby magnetic elements in our devices which might affect the magnetic environment of the Ni particles.

Future one-dimensional spin-transport devices will typically incorporate small ferromagnetic elements, and it is important to understand the resistance of these elements before the overall operation of such devices can be understood. In addition to their importance to device physics, transport measurements on ferromagnetic films provide a means of studying the magnetic properties of single domain particles over wide ranges in field and temperature which are not accessible by other measurement techniques. 
The authors wish to acknowledge extensive discussions with Anupam Garg, John Ketterson, and Yuli LyandaGeller. This work was supported by the David and Lucile Packard Foundation and the MRSEC program of the National Science Foundation (DMR-9632472) at the Materials Research Center of Northwestern University.

${ }^{1}$ M. Lederman et al., J. Appl. Phys. 73, 6961 (1993); M. Lederman et al., J. Appl. Phys. 75, 6217 (1994); M. Lederman et al., Phys. Rev. Lett. 73, 1986 (1994); R. O'Barr et al., J. Appl. Phys. 79, 5303 (1996).

${ }^{2}$ C. Salling et al., J. Appl. Phys. 75, 7989 (1994).

${ }^{3}$ W. Wernsdorfer et al., J. Magn. Magn. Mater. 145, 133
(1995).

${ }^{4}$ W. Wernsdorfer et al., Phys. Rev. Lett. 77, 1873 (1996); W. Wernsdorfer et al., Phys. Rev. B 55, 11552 (1997); W. Wernsdorfer et al., Phys. Rev. Lett. 78, 1971 (1997); W. Wernsdorfer et al., Phys. Rev. Lett. 79, 4014 (1997); W.T. Coffey et al., Phys. Rev. Lett. 80, 5655 (1998).

${ }^{5}$ C. Shearwood et al., J. Appl. Phys. 75, 5249 (1994).

${ }^{6}$ A.O. Adeyeye et al., Appl. Phys. Lett. 70, 1046 (1997).

${ }^{7}$ J.I. Martin et al., Appl. Phys. Lett. 72, 255 (1998).

${ }^{8}$ K. Hong and N. Giordano, Phys. Rev. B 51, 9855 (1995).

${ }^{9}$ K. Hong and N. Giordano, J. Phys. C. (1998).

10 T.R. McGuire and R.I. Potter, IEEE Trans. Mag. MAG11, 1018 (1975).

${ }^{11}$ Y.Q. Jia et al., J. Appl. Phys. 81, 5461 (1997).

${ }^{12}$ M. Viret et al., Phys. Rev. B 53, 8464 (1996); J.F. Gregg et al., Phys. Rev. Lett. 77, 1580 (1996).

13 T.G.S. Rijks et al., Phys. Rev. B 51, 283 (1995).

${ }^{14}$ A. Aharoni, Introduction to the Theory of Ferromagnetism [Oxford University Press, New York (1996)]. 